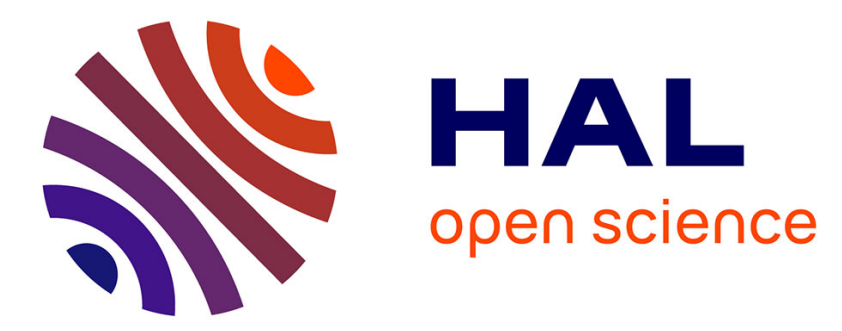

\title{
Influence of Visual Feedback on Passive Tactile Perception of Speed and Spacing of Rotating Gratings
}

\author{
Anatole Lécuyer, Marco Congedo, Edouard Gentaz, Olivier Joly, Sabine
}

Coquillart

\section{- To cite this version:}

Anatole Lécuyer, Marco Congedo, Edouard Gentaz, Olivier Joly, Sabine Coquillart. Influence of Visual Feedback on Passive Tactile Perception of Speed and Spacing of Rotating Gratings. EuroHaptics'10 2010 international conference on Haptics - generating and perceiving tangible sensations: Part II, Jul 2010, Amsterdam, Netherlands. pp.978-3-642-14074-7. inria-00537176

\section{HAL Id: inria-00537176 \\ https://hal.inria.fr/inria-00537176}

Submitted on 17 Nov 2010

HAL is a multi-disciplinary open access archive for the deposit and dissemination of scientific research documents, whether they are published or not. The documents may come from teaching and research institutions in France or abroad, or from public or private research centers.
L'archive ouverte pluridisciplinaire HAL, est destinée au dépôt et à la diffusion de documents scientifiques de niveau recherche, publiés ou non, émanant des établissements d'enseignement et de recherche français ou étrangers, des laboratoires publics ou privés. 


\title{
Influence of Visual Feedback on Passive Tactile Perception of Speed and Spacing of Rotating Gratings
}

\author{
Anatole Lécuyer ${ }^{1}$, Marco Congedo ${ }^{1,2}$, Edouard Gentaz ${ }^{3}$, \\ Olivier Joly ${ }^{4}$, Sabine Coquillart ${ }^{5}$ \\ 1. INRIA Rennes, Campus de Beaulieu, \\ 35042 Rennes Cedex, France, anatole.lecuyer@irisa.fr.
}

2. ViBS Team, GIPSA-lab CNRS, Domaine Universitaire, 38402 Saint Martin d'Hères Cedex, France, marco.congedo@gmail.com.

3. LPNC, CNRS and Université Pierre Mendès-France, 38040 Grenoble Cedex 9, France, edouard.gentaz@upmf-grenoble.fr.

4. CEA LIST, 92265 Fontenay-Aux-Roses Cedex, France, olivier.joly@cea.fr.

5. INRIA Rhône-Alpes-LIG, 655 avenue de l'Europe, Montbonnot, 38334 Saint Ismier Cedex, France, sabine.coquillart@inria.fr.

\begin{abstract}
We studied the influence of visual feedback on the tactual perception of both speed and spatial period of a rotating texture. Participants were placed in a situation of perceptual conflict concerning the rotation speed of a cylindrical texture. Participants touched a cylindrical texture of gratings rotating around its axis at a constant speed, while they watched a cylinder without gratings rotating at a different speed on a computer screen. Participants were asked to estimate the speed of the gratings texture under the finger and the spacing (or spatial period) of the gratings. We observed that the tactual estimations of both speed and spacing co-varied with the speed of the visual stimulus, although the cylinder perceived tactually rotated at a constant speed. The first effect (speed effect) could correspond to the resolution of the perceptual conflict in favor of vision. The second effect (spacing effect) is apparently surprising, since no varying information about spacing was provided by vision. However, the physical relation between spacing and speed is well established according to every day experience. Thus, the parameter extraneous to the conflict could be influenced according to previous experience. Such cross-modal effects could be used by designers of virtual reality systems and haptic devices to improve the haptic sensations they can generate using simple (constant) tactile stimulations combined with visual feedback.
\end{abstract}

Keywords: Touch, Texture, Perception, Illusion, Speed effect, Spacing effect

\section{Introduction}

Our everyday activities rely on the simultaneous and interactive involvement of different senses. The exchanges between individuals and environment are mostly multimodal. There has been increasing interest on multimodal integration and crossmodal interaction, particularly on integration of vision and touch (Hatwell, Streri and 
Gentaz, 2003; Ernst and Banks, 2002; Lécuyer, 2009). Early studies on integration of vision and touch used situations of sensory conflict and revealed a strong dominance of vision for spatial properties (Rock \& Victor, 1964). However, the notion of visual dominance has been further modulated since, because other studies have shown a "compromise" between the two conflicting values (Heller, 1983). It seems that in spatial conflicts vision is usually dominant, but tactual information comes into play abruptly when inter-modal coherence is broken. Moreover, results are different for material properties, which are the domain favored by touch. For instance, tactual perception of textures is as efficient as visual perception and sometimes, for fine textures of abrasive papers, touch surpasses vision (Heller, 1989). Contrary to what was found for spatial properties, Heller (1989) and Lederman (1974) observed no difference between active exploration (participants rub the object with their fingers) and passive one (the object is moved under the immobile fingers). Thus, perception of textures might be less the result of kinesthetic than of cutaneous information. In a conflicting situation on textures properties, with abrasive paper seen and touched, the participants gave compromise responses (Lederman \& Abbott, 1981). Their evaluation of the conflicting texture was a mean of the visual and tactual values. Lederman, Thorne and Jones (1986) dissociated two elements of textures: the notion of "roughness" (a material property) and the spatial density of the grains (a geometric property). A tactual-dominant compromise appeared when participants were instructed to estimate roughness, whereas a visual capture appeared when participants were instructed to evaluate the spatial density of the grains. Tactual dominance in the estimation of roughness was also observed in passive exploration (Guest \& Spence, 2003).

In few studies, the issue of how the perceptual influence on one parameter influences other parameters physically related to it has been addressed (Lécuyer, 2009). To further investigate this issue, we set up an unusual situation of perceptual conflict regarding the rotation speed of a cylindrical texture (a texture glued on a rotating cylinder). Participants touched (without active movements) a cylindrical texture made of gratings rotating around its axis at constant speed, while watching on a computer screen a representation of a cylinder without gratings rotating at a different speed. The rotation of the visual stimulus was sometimes largely accelerated or decelerated when compared to the actual rotation of the gratings under the finger. Participants were asked to estimate both the speed of the gratings texture under the finger, and the spacing, i.e., spatial period, of the gratings. The reviewed literature suggests that the visual perception of speed could dominate the tactual one. We then expected the tactual perception of speed to be influenced by the visual speed. We also studied the possible influence of the perceptual conflict on the tactual estimation of the spatial period of the texture.

\section{Method}

Population: Ten adults (6 men and 4 women) took part in this experiment. All participants were right-handed. 
Experimental apparatus: The texture used as tactual stimulus was a cylindrical texture of gratings (Figure 1-left). The gratings had a $1.5 \mathrm{~mm}$ height (or amplitude) and a $5 \mathrm{~mm}$ spacing (or spatial period). The cylinder on which the texture was glued had a 50mm diameter. The spacing of the gratings texture remained constant since the same texture was used along the whole experiment. The texture was set in rotation by an electrical motor. The texture was touched with the left index, perpendicularly to the axis of the cylinder and thus to the texture. The texture rotated from the interior to the exterior of the finger. The tactual stimulus was hidden to the participants' view by means of a box which enclosed both texture and participants' left hand. The left index rested on the gratings without any motion. As a consequence, the tactual perception of gratings was passive and based exclusively on cutaneous information. A small screen could be positioned between the index and the stimulus in order to enable or disable the contact between the finger and the texture. It was removed when testing the texture and immediately replaced after it.
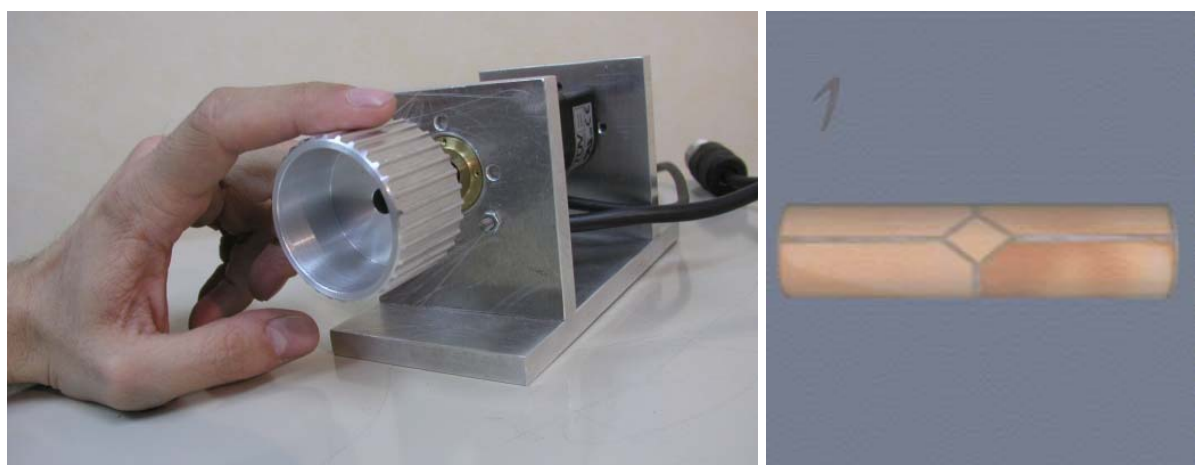

Fig. 1. Experimental apparatus: (Left) Tactual texture of gratings; (right) Visual scene.

The visual scene was made of a rotating cylinder (Figure 1-right). This cylinder had the same radius as the real cylindrical texture (i.e. $50 \mathrm{~mm}$ ) and was $10 \mathrm{~cm}$ long. The visual texture used and mapped on the cylinder on the screen was a standard image (terra cotta) provided with the O2 graphic workstation of the SGI Company. This texture was considered as "neutral", as it did not provide meaningful information in terms of spacing of the gratings. The visual stimulus was displayed on a computer screen in monoscopic conditions. The participants were seated $30 \mathrm{~cm}$ in front of the screen. The eyes of the participant were at the same height as the display of the cylinder on screen. The frame rate of the visual stimulus was of $15 \mathrm{~Hz}$.

Experimental procedure: During a test, participants were asked to touch the tactual stimulus with their left index while looking at the computer screen. Participants were told that the cylinder displayed on the computer screen was a representation of the rotating shaft on which their finger rested. We used the magnitude estimation method (Lederman, Thorne \& Jones, 1986). During each trial, participant began to perceive the speed and spacing of a first texture (reference condition). After a 2-second break, they were asked to perceive the speed and spacing of a second texture (comparison condition). Participants were allowed unlimited time needed to evaluate each texture, 
but they were asked to perform the trials as quickly as possible. After each trial, the participants were asked to grade both the speed and the spacing of the comparison texture located under the finger, as compared to the reference texture, using a 10based scale. For example, a speed -or spacing- of the comparison texture estimated as two times faster -or wider- was graded with a 20 value. For the purpose of the analysis, the estimations can be converted into rpm values for the speed and $\mathrm{mm}$ values for the spacing.

The spacing of the gratings remained constant throughout the experiment and equal to $5 \mathrm{~mm}$. The rotation speed of the texture remained also constant under the finger and equal to 15rpm for both the reference and the comparison conditions. The linear speed of the texture at the surface of the finger was thus equal to $39.27 \mathrm{~mm} / \mathrm{s}$. In the reference condition, the visual speed of the cylinder on the computer screen was also equal to 15rpm. In the comparison condition, 7 different visual speeds were used (smaller, equal or greater than the tactual one): 7.5, 10.5, 13.5, 15, 16.5, 19.5, and $22.5 \mathrm{rpm}$. Each comparison pair was tested 4 times, for a total of $4 \times 7=28$ trials per participant. The 7 comparison pairs were presented randomly. The experiment lasted around 40 minutes. The participants wore earphones to eliminate noise cues.

\section{Results}
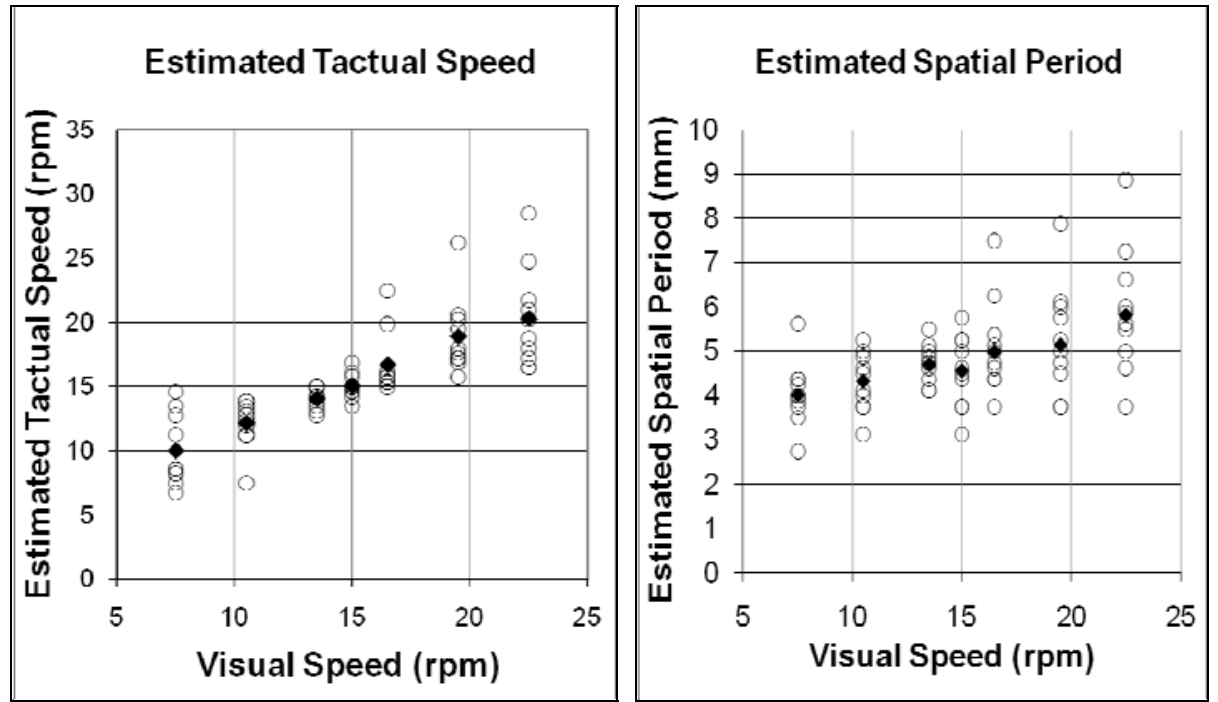

Fig. 2. Experimental results (black circle = one participant, black rhombus = average): (Left) Estimated tactual speed of the texture ; (Right) Estimated spatial period of the texture.

Estimation of Speed: Figure 2-left shows individual estimations of the tactual speed averaged across the four blocks of trials. The fitted line with all individual estimations $(\mathrm{N}=70)$ has equation $y=6.87 \mathrm{x}+0.61$. The $\mathrm{R}$-squared value is 0.53 . The t-test for the slope being different from zero is significant $(\mathrm{t}(68)=8.75 ; \mathrm{p}<0.0001)$. The estimated tactual speed seems thus positively correlated to the visual speed. 
Estimation of Spatial Period: Figure 2-right shows individual estimations of the spatial period averaged across the four blocks $(\mathrm{N}=70)$. With spatial period data converted to rpm values, so to allow comparison with results obtained with Speed estimation, the obtained fitted line has equation $y=4.68 x+0.71$. The R-squared value is 0.67 . The $t$-test for the slope being different from zero is significant $(t(68)=11.74$; $\mathrm{p}<0.0001)$. The estimated spacing is positively correlated to the visual speed. Thus, the tactual estimates of the spatial period of the texture seem also influenced by the speed of the visual stimulus.

\section{Perspectives}

Summary of results: Our studies explored two cross-modal effects of vision on tactual perception. The first effect, named the "speed effect", concerned the visuohaptic perceptual estimation of the rotation speed of a cylinder. All participants reported a change in the tactual speed although this speed remained constant during all trials. If the visual and tactual percepts do not agree, the visual feedback was found to influence the tactual perception of the constant stimulus. The second effect, named the "spacing effect", concerned the estimation of the spatial period (spacing) of the gratings texture located on the cylinder. When the visual speed was greater than the real one, the participants reported that the spacing of gratings increased, and when the visual speed was smaller than the real one they reported that the spacing decreased. In this case, no relevant information about spacing was provided by the visual stimulus, but the tactual estimation of spacing was still influenced as for speed. Therefore this experiment shows that when touching gratings which rotate at a constant speed, the tactual perception of both the speed and spatial period of the gratings is influenced by the visual perception of the rotation speed of a "neutral" texture rotating at different speeds. The speed and spatial period of the texture perceived tactually tend to increase (or decrease) when the rotation of the visual stimulus is accelerated (or decelerated). Indeed, the varying visual stimuli influence the perception of the two constant tactual parameters. A relation between stimuli coming from separate modalities is built by the participants, and question of how this phenomenon operates remains open.

Discussion: The speed effect is in line with previous research showing that for spatial properties vision dominates touch in the bimodal integration process. The spacing effect is more surprising. No explicit relation existed in the experimental materials between the parameter estimated tactually and the visual stimulus. There was indeed no relevant information available on the visual scene to provide a notion of spatial period of the gratings. However, a cross-modal association is arbitrarily established between the two stimuli, as shown by Figure 2. An interpretation of the spacing effect consists in considering a texture of rectangular gratings moving at the surface of a finger. A relation does exist between three parameters: the speed of the texture, its spatial period (spacing) and the resulting temporal frequency which corresponds to the temporal activation of the surface of the finger at a single point (Cascio \& Sathian, 2001). This relation ensures the physical homogeneity and is given by Equation 1, 
where $\mathrm{P}$ (in $\mathrm{mm}$ ) is the density or spatial Period of the texture (in our case: the spacing of the gratings), $\mathrm{S}(\mathrm{mm} / \mathrm{s})$ is the Speed of the texture at the level of the finger, and $\mathrm{F}(\mathrm{Hz}$, or $\mathrm{s}-1)$ is the temporal Frequency sensed at the level of the finger and induced by the uniform motion of the texture under the finger. Thus, $\mathrm{F}$ is the number of consecutive activations at a single point or at a single cutaneous mechanoreceptor. The cross-modal association made by the participants could stem from the physical relation implied by Equation 1.

$$
\mathrm{P}=\mathrm{S} / \mathrm{F}
$$

Applications: The two cross-modal effects demonstrated here could be of great interest for the designers of haptic devices and virtual reality systems. Our results suggest indeed that the use of a constant haptic (tactile) stimulus can be combined with varying visual stimuli to generate a wide range of haptic sensations. This implies new uses and requirements for the design of cheap and simple tactile peripherals, augmented by visual feedback.

\section{References}

1. Cascio, C.J., \& Sathian, K. (2001). Temporal cues contribute to tactile perception of roughness. Journal of Neuroscience, 21, 5289-5296.

2. Ernst, M.O., \& Banks, M.S. (2002). Humans integrate visual and haptic information in a statistically optimal fashion. Nature, 415, 429-433.

3. Guest, S., \& Spence, C. (2003). Tactile dominance in speeded discrimination of textures. Experimental Brain Research, 150, 201-207.

4. Hatwell, Y., Streri, A., \& Gentaz, E. (2003). Touching for knowing. Amsterdam/Philadelphia: John Benjamins Publishing Compagny.

5. Heller, M. A. (1983). Haptic dominance in form perception with blurred vision. Perception, 12, 607-613.

6. Heller, M. A. (1989). Texture perception in sighted and blind observers. Perception and Psychophysics, 45, 49-54.

7. Lécuyer, A. (2009). Simulating Haptic Feedback using Vision: a Survey of Research and Applications of Pseudo-Haptic Feedback. Presence: Teleoperators and Virtual Environments, 18(1), 39-53.

8. Lederman, S. J. (1974). Tactile roughness of grooved surfaces: The touching processes and effects of macro and microsurface structure. Perception and Psychophysics, 16, 385396.

9. Lederman, S.J., \& Abbott, S.G. (1981). Texture perception: Studies of intersensory organization using a discrepancy paradigm, and visual versus tactual psychophysics. Journal of Experimental Psychology: Human Perception and Performance, 7, 902-915.

10. Lederman, S.J., Thorne, G., \& Jones, B. (1986). Perception of texture by vision and touch: Multidimentionality and intersensory integration. Journal of Experimental Psychology: Human Perception and Performance 12, 169-180.

11. Rock, I., \& Victor, J. (1964). Vision and touch: An experimentally created conflict between the two senses. Science, 143, 594-596. 